\title{
Learning Analytics em Ação: Uma Revisão Sistemática de Literatura
}

\author{
Elyda Laisa Soares Xavier Freitas ${ }^{1,2}$, Fernando da Fonseca de Souza ${ }^{1}$, Vinicius \\ Cardoso Garcia ${ }^{1}$
}

${ }^{1}$ Centro de Informática - Universidade Federal de Pernambuco (UFPE)

Caixa Postal 15.064 - 91.501-970 - Recife - PE - Brasil

${ }^{2}$ Instituto de Informática - Universidade de Pernambuco (UPE) - 55002-971 - Caruaru PE - Brasil

\{elsx, fdfd, vcg\}@cin.ufpe.br

\begin{abstract}
Context: Learning Analytics (LA) is a recent trend in education, and its impacts are not widely demonstrated yet. Aim: Identify and summarize practical results of using LA concerning teaching and learning. Methodology: Systematic Literature Review. Results: From 757 papers, 14 have shown evidence to understand practical results for both teachers and students while using LA. Conclusions: LA has contributed to improve teaching and learning in different circumstances, after students receiving automated feedback, as well as has supported teachers to achieve insights about assessments, which could never be revealed without using $L A$.
\end{abstract}

Resumo. Contexto: Learning Analytics (LA) é uma tendência recente na educação e os impactos de sua adoção ainda não estão amplamente demonstrados. Objetivo: Identificar e sumarizar os resultados práticos da utilização de LA no que se refere ao ensino e aprendizagem. Metodologia: Revisão Sistemática de Literatura. Resultados: De um total de 757 artigos, 14 apresentaram evidências que permitiram entender os resultados práticos do uso de LA para professores e estudantes. Conclusão: LA contribuiu para melhorar o ensino e aprendizagem em diferentes circunstâncias, após os estudantes receberem feedbacks automatizados, e apoiou os instrutores a alcançarem percepções que, sem o apoio de LA, sequer seriam conhecidas.

\section{Introdução}

Learning Analytics (LA) é a disciplina que analisa dados educacionais obtidos a partir da interação do estudante com recursos online [Blanco et al. 2013]. Ela se utiliza dos dados disponíveis nos Sistemas de Gestão de Aprendizagem (do inglês Learning Management Systems, LMS) - bem como em outras fontes - para realizar análises que auxiliam o aluno e o professor a compreender e avaliar o processo de aprendizagem, permitindo a tomada de decisões. LA não serve apenas aos objetivos das instituições de ensino mas também pode ser direcionada a outras partes interessadas [Chatti et al. 2012]. Aos estudantes, permite aumentar a motivação e ajudar na autorreflexão sobre os seus processos de aprendizagem [Santos et al. 2012]. Aos professores, pode prover insumos para auxílio aos alunos e avaliação de objetos de aprendizagem [Dyckhoff et 
al. 2012]. LA informa e dá suporte ao processo de aprendizagem do estudante, prometendo melhorá-lo [Yassine et al. 2016; Siemens e Baker 2012]. Ademais, motiva mudanças que vão desde a conduta do estudante com base em informações sobre sua aprendizagem até as práticas pedagógicas dos professores.

Apesar das vantagens presumíveis pelo uso de Learning Analytics, poucas publicações apresentam resultados práticos consistentes, obtidos pelo uso contínuo das ferramentas ou em contato real com os alunos, por meio dos quais seria possível avaliar os efeitos obtidos pelas instituições de ensino. Conforme afirmam [Liu et al. 2016], é preciso "preencher a lacuna entre capacidade tecnológica e melhorias tangíveis da experiência do estudante". Diante desse contexto, este artigo visa identificar relatos de experiências que permitam conhecer as consequências práticas da aplicação de LA, de modo a entender seu impacto sobre os processos de ensino e aprendizagem, a fim de ratificar os benefícios prenunciados bem como a solidez dos resultados dessa disciplina.

\section{Metodologia}

Para a realização desta pesquisa optou-se pelo método de Revisão Sistemática de Literatura (RSL), conforme guia descrito em [Kitchenham e Charters 2007], e que é definido pelos autores como "um meio de avaliar e interpretar toda pesquisa relevante disponível com relação a uma questão de pesquisa, área, ou fenômeno de interesse". Isto é, a RSL visa extrair apenas a informação útil de artigos científicos publicados a fim de atender aos objetivos da pesquisa. O propósito da RSL nesta pesquisa é "sumarizar a evidência existente com relação a um tratamento ou tecnologia (...)" [Kitchenham e Charters 2007]. Ou seja, sintetizar as evidências de aplicação de LA nas instituições, descrevendo seus resultados práticos para o ensino e aprendizagem. Esta seção apresenta o planejamento desta pesquisa por meio de seu protocolo.

\subsection{Pergunta de Pesquisa}

A seguinte pergunta de pesquisa foi definida: Como Learning Analytics tem sido aplicada para apoiar o ensino e aprendizagem? Ou seja, esta pesquisa objetiva identificar a adoção de LA nas instituições e seus resultados práticos. Para apoiar a análise de dados, as seguintes sub-questões foram definidas: (a) Quais as ferramentas/técnicas utilizadas? e (b) De que modo o uso de LA melhorou o ensino e aprendizagem na instituição? Dada a extensão dos resultados obtidos, o presente artigo contempla exclusivamente a sub-questão de pesquisa (b).

\subsection{Estratégia de Busca dos Estudos Primários}

Para alcançar o conjunto mais extenso e relevante de estudos, as seguintes decisões guiaram a RSL:

- Fontes de Informação - Optou-se por utilizar o engenho de busca Scopus, dada sua relevância, visto que abrange publicações de alta qualidade de periódicos e anais de congressos.

- Estratégia de Busca - Uma busca automática foi realizada e apenas artigos científicos revisados por pares foram considerados. A busca foi conduzida em etapas, as quais estão descritas a seguir. 
VIII Congresso Brasileiro de Informática na Educação (CBIE 2019)

Anais do XXX Simpósio Brasileiro de Informática na Educação (SBIE 2019)

- Equipe - A equipe de busca foi composta por duas pessoas. Além disso, um terceiro membro ficou responsável por resolver questões de empate nas discussões. A seguinte metodologia foi utilizada para a realização da RSL: Cada pesquisador ficou responsável por realizar a busca no Scopus. Os pesquisadores realizaram a pesquisa no engenho e aplicaram o primeiro filtro, lendo o título e o resumo. Quaisquer conflitos relativos à inclusão ou à exclusão de um artigo foram decididos pela sua inclusão em um processo de união de conjuntos. Em seguida, os dois pesquisadores avaliaram esse resultado, lendo o conjunto de artigos e aplicando os critérios de inclusão e exclusão (apresentados a seguir). Nesse momento, os conflitos não podem persistir, de modo que um terceiro membro decide pela inclusão ou não do artigo.

- $\quad$ String de Busca - Com base nas perguntas de pesquisa, a seguinte estrutura PIO foi definida: População (P): Teaching or Learning; Intervenção (I): Techniques and Tools for LA; Resultado (O): ? . Assim, a seguinte string de busca foi utilizada, tendo sido adicionados os sinônimos: ( "technique" OR "method" OR "performance" OR "approach" OR "practice" OR "manner" OR "procedure") AND ("application" OR "tool" OR "implementation") AND ("teaching" OR "learning") AND ("learning analytics")).

- Critérios de Inclusão e Exclusão - A inclusão de um artigo é definida pela sua relevância em responder à questão de pesquisa, a ser determinada pela análise do artigo completo. Os seguintes critérios de inclusão foram definidos: (a) Estudos cujo tema é Learning Analytics; (b) Estudos que apresentam execuções práticas de LA: as aplicações devem ser testadas em ambientes reais e devem expor resultados concretos do uso de LA sobre o ensino e/ou aprendizagem. Os critérios de exclusão definidos foram: (c) artigos que não estejam em língua inglesa ou portuguesa; (d) Artigos de Gray Literature; (e) Estudos não disponíveis em formato digital; (f) Short Papers com menos de 6 páginas - em razão da necessidade de exposição clara e detalhada dos métodos e resultados do artigo; e (g) Estudos disponíveis apenas em bibliotecas pagas as quais a equipe não tem livre acesso. Se um artigo sob avaliação se enquadra simultaneamente nos dois critérios de inclusão (a) e (b), este será considerado incluído. Por outro lado, se o artigo se enquadra em pelo menos um critério de exclusão, este será classificado como excluído.

\section{Execução da Pesquisa}

A string de busca foi executada no engenho Scopus no dia 25 de abril 2019, resultando em 757 artigos encontrados. Os seguintes critérios foram utilizados: a busca deve ser realizada no título, resumo e palavras-chave, o período definido foi de 2011 a 2018 e os tipos de documento foram "Conference Paper" e "Article".

Após a leitura do título e resumo, o total de artigos foi reduzido para 325 (destes, $15,6 \%$ estão em bases de dados científicas pagas, as quais não estavam disponíveis ao grupo de pesquisa). A aplicação dos critérios de inclusão e exclusão resultou num total de 14 artigos. As razões mais recorrentes para a exclusão de um artigo foram os critérios (b) e (f) $(66,5 \%$ e $25 \%$, respectivamente). Também foram excluídos artigos devido aos critérios (c), (a) e (d). Os artigos selecionados foram 
VIII Congresso Brasileiro de Informática na Educação (CBIE 2019)

Anais do XXX Simpósio Brasileiro de Informática na Educação (SBIE 2019)

publicados no período de 2013 a 2018, sendo a maior parte deles em 2018 (35,7\%). Desses artigos, $65 \%$ foram publicados em conferências e $35 \%$ em periódicos.

\section{Resultados}

Durante a execução desta pesquisa, identificou-se a existência de uma grande variedade de ferramentas que aplicam os conceitos de Learning Analytics. No entanto, muitos trabalhos apenas descreviam as funções da ferramenta desenvolvida; outros utilizavamse de bases de dados sobre os estudantes relativas a anos anteriores com o intuito de validar as ferramentas ou métodos propostos; uma terceira categoria de trabalhos destacava a utilização de ferramentas mas não avaliava de forma alguma o resultado de sua utilização. Vale ressaltar que todos esses artigos foram excluídos por não atenderem ao critério de inclusão (b), uma vez que não apresentavam evidências empíricas que demonstrassem as consequências reais do uso de LA para alunos e professores. Dessa forma, apenas $1,84 \%$ dos artigos atendeu aos critérios definidos para a pesquisa.

$\mathrm{Na}$ análise desses artigos foram identificadas diferentes experiências reportando o uso de Learning Analytics. Dentre esses resultados, há iniciativas que constataram melhora do desempenho dos estudantes, maior suporte para os professores em suas atividades de avaliação, além de outras experiências nas quais os estudantes relatam que a ferramenta foi útil para ajudar na reflexão sobre suas atividades. Cada um desses projetos está sintetizado nas seções seguintes.

\section{1. "Mining Student Repositories to Gain Learning Analytics: An Experience Report” [Robles e Gonzalez-Barahona 2013]}

Esse trabalho aplicou técnicas de Mining Software Repositories (MSR) a fim de avaliar atividades de programação enviadas pelos estudantes de um curso de Redes Multimídia. $\mathrm{O}$ artigo descreve um estudo realizado em dois cursos que utilizaram uma ferramenta capaz de avaliar se o programa enviado atende aos critérios de avaliação definidos pelo professor, provendo feedback detalhado aos estudantes sobre a análise, sendo capaz também de identificar a ocorrência de plágio, visto que o programa pode criar testes personalizados para cada estudante com base no código enviado por ele. Como resultado do estudo, verificou-se que os estudantes utilizaram o feedback para promover melhorias nos códigos, com progressos notáveis a cada entrega. Além disso, passaram a utilizar de forma mais eficiente os comentários no sistema de controle de versões, tornaram-se mais conscientes dos benefícios do versionamento e entenderam com maior antecedência no semestre os conceitos sobre redes de computadores, dentre outros benefícios.

\section{2. "PolyCAFe-Automatic Support for the Polyphonic Analysis of CSCL Chats" [Trausan-Matu et al. 2014]}

Utilizando técnicas de Processamento de Linguagem Natural, Social Network Analysis e Information Retrieval, o PolyCAFe System realiza a análise de conversações de pequenos grupos em chats ou fóruns de discussão, dando feedback aos estudantes e tutores sobre o desempenho individual e em grupo na resolução de tarefas dadas pelo tutor, com base no envolvimento e impacto das participações. Foram realizados dois ciclos de testes, sendo o primeiro um estudo piloto. Um total de 35 estudantes participou do experimento (sendo 10 no grupo de controle). Como resultado do 
VIII Congresso Brasileiro de Informática na Educação (CBIE 2019)

Anais do XXX Simpósio Brasileiro de Informática na Educação (SBIE 2019)

experimento, os seguintes resultados foram obtidos: (1) os testes mostraram, e os tutores confirmaram, uma redução de 35\% no tempo médio necessário para avaliar uma dada atividade, comparando ao tempo necessário para avaliação sem o uso da ferramenta PolyCAFe; (2) os estudantes confirmaram a acurácia, relevância e consistência do feedback (com concordância de 60 a 80\%). Ademais, os estudantes demonstraram utilização intensiva da ferramenta durante o período de avaliação; e (3) dentre os tópicos avaliados pela ferramenta, houve uma melhora nos quesitos expressão e colaboração $(6,8 \%$ e $29 \%$, respectivamente, no segundo chat em comparação ao grupo de controle). Além disso, em comparação às avaliações realizadas pelos tutores e demais participantes do chat, a ferramenta se mostrou com alta precisão para atribuição de notas individuais.

\section{3. "Open Student Models of Core Competencies at the Curriculum Level: Using Learning Analytics for Student Reflection” [Chou et al. 2017]}

$\mathrm{O}$ artigo reporta a utilização da ferramenta VACC (Visualized Analytics of Core Competencies, em português, Análise Visual de Competências Essenciais), cujo propósito é apoiar a reflexão dos estudantes sobre competências essenciais e disciplinas cursadas. A partir da análise do histórico dos alunos é gerado um diagnóstico, identificando quais competências essenciais o estudante satisfaz ou não - apontando a correspondência entre disciplinas cursadas e competências alcançadas. A ferramenta permite ainda que o estudante compare seu desempenho com os colegas e também que identifique disciplinas disponíveis para melhorar as competências que desejar. Em um experimento com 155 estudantes, estes avaliaram que a ferramenta foi útil para ajudálos a entender sua situação atual $(+70 \%)$ e a planejar as próximas disciplinas a serem cursadas $(72 \%)$.

\section{4. "Learning Analytics on Graduate's Academic Records to Reflect on a Competency-Based Curriculum" [Chou et al. 2018]}

Também fazendo uso do sistema VACC, esse estudo visa apoiar comitês de avaliação de programas de ensino na reflexão sobre objetivos e competências do programa, deficiências no ensino e também no aprendizado do estudante. A ferramenta ajuda a identificar quais competências têm sido negligenciadas em programas e quais são exploradas excessivamente, além de ajudar a propor um conjunto mínimo de competências desejadas para o currículo. É possível também ter uma visão geral do desempenho dos estudantes em cada competência. O VACC está em uso na instituição desde 2013, em 14 departamentos, e seu uso motivou revisões no currículo com base nas informações obtidas - as quais são relatadas em detalhes para cada departamento no artigo original. Dentre as mudanças sugeridas, por exemplo, algumas competências foram removidas dos cursos de Ciência da Computação e Engenharia. Além disso, o departamento conseguiu diminuir em 31\% a diferença de desempenho entre os 50\% melhores e 50\% piores com a obrigatoriedade de participação de estudantes em situação de risco em grupos de estudos. Outro exemplo relatado foi o departamento de Ciências Sociais e Políticas, o qual ajustou disciplinas entre eletivas e obrigatórias para balancear as competências que se desejava que o estudante alcançasse. 
VIII Congresso Brasileiro de Informática na Educação (CBIE 2019)

Anais do XXX Simpósio Brasileiro de Informática na Educação (SBIE 2019)

\section{5. "Using Test Data for Successive Refinement of an Online Pre-Course in Mathematics” [Derr et al. 2015]}

O objetivo desse trabalho foi estabelecer e refinar um pré-curso de Matemática para estudantes de Engenharia. Learning Analytics, utilizada em conjunto com métodos qualitativos, permitiu a identificação das mudanças necessárias para que o curso atendesse aos estudantes de maneira mais adequada. Uma das ações resultantes da análise dos dados foi o agrupamento dos estudantes em turmas de acordo com o seu rendimento. Verificou-se melhora no desempenho dos estudantes após a participação nesse curso.

\section{6. "Student Data: Data is Knowledge - Putting the Knowledge Back in the Students' Hands" [Corrigan et al. 2015a] e "Using Educational Analytics to Improve Test Performance” [Corrigan et al. 2015b]}

Os dois artigos são dos mesmos autores e descrevem um projeto no qual os estudantes receberam feedbacks semanais sobre a previsão de seu desempenho em determinado módulo com base nos seus resultados atuais. É importante ressaltar que não foram identificadas diferenças prévias significativas nas notas dos estudantes dos grupos de controle e de experimento. Por fim, os estudantes que participaram do experimento tiveram um desempenho em média $3 \%$ superior nos exames com relação àqueles que optaram por não participar. Além disso, na avaliação qualitativa descrita no primeiro artigo, 33\% dos estudantes relataram que mudaram seu comportamento devido aos feedbacks recebidos, inclusive tendo se esforçado para estudar mais.

\section{7. "Empowering Instructors through Customizable Collection and Analyses of Actionable Information” [Liu et al. 2016]}

Esse trabalho reporta as consequências do desenvolvimento de uma ferramenta para Learning Analytics cujo objetivo é melhorar a interação por meio do engajamento e comunicação. A ferramenta é customizável e permite que os instrutores especifiquem condições para identificar estudantes em grupos a fim de enviar feedbacks sobre o seu desempenho via e-mail ou celular. O sistema já foi usado por mais de 20.000 alunos. As análises geradas pela ferramenta identificaram que o desempenho dos estudantes em questionários é um importante fator de sucesso dos mesmos. Assim, foram propostas mudanças pedagógicas, como a provisão de feedback nesses questionários, a fím de melhorar o desempenho dos estudantes.

\section{8. "Reflective Writing Analytics for Actionable Feedback" [Gibson et al. 2017]}

Nesse projeto, Learning Analytics foi utilizada para avaliar a escrita dos estudantes, fornecendo feedbacks para reflexão e consequente melhoria na qualidade dos textos. Depoimentos reflexivos enviados pelos estudantes foram analisados semanalmente e o feedback apresentado pela ferramenta foi avaliado como útil pelos estudantes $(85,7 \%)$. Além disso, foram identificados textos corrigidos pelos estudantes após o feedback, cuja melhora na qualidade foi verificada com clareza. 
VIII Congresso Brasileiro de Informática na Educação (CBIE 2019)

Anais do XXX Simpósio Brasileiro de Informática na Educação (SBIE 2019)

\section{9. "Teamwork Assessment in the Educational Web of Data: A Learning Analytics Approach Towards ISO 10018” [Conde et al. 2018]}

Esse artigo expõe o uso de uma ferramenta de LA para apoiar o professor na avaliação de competências relacionadas ao trabalho em grupo. No estudo de caso, foram propostas atividades a serem solucionadas em equipe, usando principalmente o fórum, e publicando os resultados da atividade numa wiki. Por meio da análise dessas mensagens foi possível identificar o comportamento das equipes e atribuir notas individuais e ao grupo. $\mathrm{Na}$ análise qualitativa, os professores apontaram a ferramenta como um apoio importante para a avaliação dos grupos, especialmente no que se refere à redução de tempo e esforço para avaliação.

\subsection{0. "WebIntera-Classroom: an Interaction-Aware Virtual Learning Environment for Augmenting Learning Interactions" [Chen et al. 2017]}

WebIntera-Classroom é um Ambiente Virtual de Aprendizagem que provê diversas ferramentas que visam aumentar as interações entre os estudantes e o conteúdo e entre estudantes e instrutores. Nesse projeto, LA é utilizada para identificar pontos cruciais de aulas gravadas, informando aos alunos que eles devem prestar mais atenção a esses momentos. Além disso, as informações sobre as interações dos estudantes com o material são repassadas aos instrutores em um dashboard a fim de fundamentar decisões pedagógicas. Os resultados demonstraram que pelo uso da ferramenta, comparado às aulas tradicionais, os estudantes puderam alcançar uma melhora no desempenho.

\subsection{1. "The Teacher in the Loop: Customizing Multimodal Learning Analytics form Blended Learning” [Rodríguez-Triana et al. 2018]}

Nesse trabalho foi utilizada uma ferramenta de LA que permite a avaliação do estudante quanto a desvios do cenário desejado com base em critérios como participação, uso dos recursos, colaboração, entre outros; e cujo desenvolvimento foi realizado com a participação dos instrutores. $O$ uso da ferramenta trouxe ganhos no tocante à sensibilidade para identificar desvios de rota - os professores relataram que em muitos casos não haviam percebido tais situações até que fossem informados por meio da ferramenta - e à diminuição do tempo de avaliação dos estudantes, permitindo verificações antes impossíveis dado o tempo necessário para tal. Além disso, a participação dos professores no processo de desenvolvimento trouxe maior confiança nos seus resultados. Por fim, os autores também propõem um método para quantificar o benefício da adoção da solução de LA para os professores.

\subsection{2. "Real-Time Learning Analytics System for Improvement of on-site Lectures"} [Shimada et al. 2018]

A ferramenta utilizada nesse trabalho realiza análises a fim de prover feedback aos professores com o objetivo de melhorar as aulas. Antes do encontro, o professor tem acesso a informações de quais páginas do material de aula não foram bem compreendidas pelos estudantes, com base em um teste respondido por eles após a leitura do material. Já durante a aula, o feedback em tempo real ajuda o professor a sincronizar a velocidade de leitura do material, seguindo o ritmo da turma, permitindo aos estudantes realizarem mais anotações sobre a aula e sobre o material. Mais uma 
VIII Congresso Brasileiro de Informática na Educação (CBIE 2019)

Anais do XXX Simpósio Brasileiro de Informática na Educação (SBIE 2019)

vez, os testes realizados com um grupo experimental e outro de controle mostraram uma maior sincronia na leitura durante as aulas do primeiro grupo. Além disso, o comportamento/ estratégia do professor mudou de uma turma a outra a fim de atender melhor aos estudantes levando em conta o feedback recebido na ferramenta (resultando num total de cinco casos de melhorias identificados).

\subsection{3. "Learning Analytics for Interpreting” [Taibi et al. 2018]}

Por fim, esse trabalho permite a análise do comprometimento e conquistas dos estudantes na criação de glossários para melhoria do vocabulário. O feedback sobre as avaliações - e sobre os que mais precisavam de ajuda - foi dado pessoalmente e, depois disso, alguns estudantes em particular mudaram seu comportamento, estimulados a construir e expandir seus glossários.

\section{Discussão}

Os trabalhos avaliados têm objetivos diversos, dentre os quais: avaliar o estudante (42\%), avaliar currículos dos programas ou atividades desempenhadas pelos estudantes (21\%), dar feedback ao estudante sobre o seu desempenho (14\%), prever o desempenho dos estudantes (14\%) e, por fim, ajudar o estudante a autorregular seu aprendizado (7\%). Diversas fontes de dados foram utilizadas nos trabalhos avaliados, tais como os próprios Sistemas de Gestão de Aprendizagem (chats, fóruns ou notas, por exemplo) [Trausan-Matu et al. 2014; Conde et al. 2018], dados de repositórios de software [Robles e Gonzalez-Barahona 2013], de ferramentas desenvolvidas especialmente para os experimentos [Chou et al. 2017; Shimada et al. 2018], também dados demográficos e de histórico dos alunos [Derr et al. 2015], bem como de navegação dos usuários [Taibi et al. 2018].

A análise dos artigos selecionados permitiu identificar com clareza como LA colaborou para o progresso do ensino e aprendizagem, bem como sua influência na avaliação e aperfeiçoamento das estruturas curriculares de diferentes cursos. Considerando a sub-questão de pesquisa (b) ("De que modo o uso de LA melhorou o ensino e aprendizagem na instituição?"), constatou-se que com o uso das ferramentas foram registradas melhorias na qualidade das atividades dos estudantes, no desempenho dos estudantes que receberam feedbacks sobre seu rendimento, no apoio aos estudantes na decisão sobre quais disciplinas cursar no semestre, entre outros. No que se refere ao ensino, as ferramentas apoiaram o professor na avaliação das atividades e dos estudantes (individualmente ou em grupo), diminuindo o tempo e esforço dedicados para essa função e permitindo, consequentemente, mais tempo para que professores se dedicassem a outras questões relacionadas ao ensino. Alguns professores relataram que não seria possível realizar certas avaliações providas pelas ferramentas de LA caso fosse realizada uma verificação manual das ações de cada aluno. Foram identificadas também mudanças no comportamento de professores e estudantes, tendo em vista as informações disponíveis.

Apesar dos resultados positivos identificados, a RSL realizada mostrou que grande parte dos trabalhos publicados até então não apresentou resultados que demonstrem como Learning Analytics deu suporte ao ensino e aprendizagem em situações reais, dado que esta é a sua principal função. Tal constatação confirma que as 
VIII Congresso Brasileiro de Informática na Educação (CBIE 2019)

Anais do XXX Simpósio Brasileiro de Informática na Educação (SBIE 2019)

pesquisas na área ainda encontram-se em fase inicial e que se faz necessário encaminhar esforços futuros das pesquisas para uma nova direção, de investigações mais profundas, a fim de que LA possa alcançar uma maior maturidade e, por consequência, maior popularidade.

\section{Conclusões e Limitações da Pesquisa}

A execução da Revisão Sistemática de Literatura confirmou o que foi anteriormente identificado em estudos preliminares, de que o registro de melhorias efetivas obtidas pela aplicação da disciplina de Learning Analytics ainda é escasso. Dos 757 artigos obtidos pela execução da string de busca, apenas 14 foram selecionados após aplicação rigorosa do método de pesquisa. Foram identificados relatos de benefícios ao ensino e aprendizagem pela aplicação de LA, havendo ainda percepções alcançadas pelos professores que, sem o apoio de LA, sequer seriam conhecidas. Uma limitação identificada nesta pesquisa é a impossibilidade de acesso a 51 artigos $(15,6 \%)$, inseridos após a leitura de título e resumo, mas que estavam em bases científicas indisponíveis ao grupo de pesquisa. No entanto, uma quantidade representativa de artigos foi analisada e os resultados apresentados permitiram responder satisfatoriamente à pergunta de pesquisa.

\section{Referências}

Blanco, Á., Serrano, Á., Freire, M., Martínez-Ortiz, I. and Fernández-Manjón, B. (2013) "E-Learning Standards and Learning Analytics. Can Data Collection Be Improved by Using Standard Data Models?" In IEEE Global Engineering Education Conference EDUCON, 13-15 March, Berlin, Germany, p. 1255-1261.

Chatti, M. A., Dyckhoff, A. L., Schroeder, U. and Thüs, H. (2012) “A reference model for learning analytics”. In Int. J. of Tech. Enhanced Learning, v.4, n.5,6, p. 318-331.

Chen, J., Xu, J., Tang, T. and Chen, R. (2017) "WebIntera-Classroom: An InteractionAware Virtual Learning Environment for Augmenting Learning Interactions". In Interactive Learning Environments, v.25, n.6, p. 792-807.

Chou, C. et al. (2017) "Open Student Models of Core Competencies at the Curriculum Level: Using Learning Analytics for Student Reflection". In IEEE Transactions on Emerging Topics in Computing, v. 5, n. 1, p. 32-44.

Chou, C. et al. (2018) "Learning analytics on graduates' academic records to reflect on a competency-based curriculum". In Computer Applications in Engineering Education, v. 26, n. 6, p. 2168-2182.

Conde, M. A., Colomo-Palacios, R., García-Peñalvo, F. J. and Larrucea, X. (2018) "Teamwork assessment in the educational web of data: A learning analytics approach towards ISO 10018”. In Telematics and Informatics, v. 35, n. 3, p. 551-563.

Corrigan, O., Glynn, M., McKenna, A., Smeaton, A. and Smyth, S. (2015a) "Student data: data is knowledge - putting the knowledge back in the students' hands". In 14th European Conf. on e-Learning ECEL, 29-30 Oct, Hatfield, UK, p. 165-172.

Corrigan, O., Smeaton, A. F., Glynn, M. and Smyth, S. (2015b) "Using educational analytics to improve test performance". In 10th European Conference on Technology Enhanced Learning EC-TEL, 15-18 Sept, Toledo, Spain, p.1-14. 
VIII Congresso Brasileiro de Informática na Educação (CBIE 2019)

Anais do XXX Simpósio Brasileiro de Informática na Educação (SBIE 2019)

Derr, K., Hübl, R. and Ahmed, M. (2015) "Using Test Data for Successive Refinement of an Online Pre-Course in Mathematics". In 14th European Conference on eLearning ECEL, 29-30 Oct, Hatfield, UK, p. 173-180.

Dyckhoff, A. L., Zielke, D., Bültmann, M., Chatti, M. A., and Schroeder, U. (2012). "Design and Implementation of a Learning Analytics Toolkit for Teachers". In Educational Technology \& Society, v. 15, n.3, p. 58-76.

Gibson, A. et al. (2017) "Reflective writing analytics for actionable feedback". In 7th International Learning Analytics \& Knowledge Conference LAK, 13-17 March, Vancouver, Canada, p. 153-162.

Kitchenham, B. and Charters, S. (2007) "Guidelines for performing Systematic Literature Reviews in Software Engineering”, v. 2.3. Tech. Report, EBSE-2007-01.

Liu, D. Y. T., Taylor, C. E., Bridgeman, A. J., Bartimote-Aufflick, K., and Pardo, A. (2016) "Empowering instructors through customizable collection and analyses of actionable information”. In CEUR Workshop Proceedings, v. 1590, p. 3-9.

Robles G. and Gonzalez-Barahona, J. M. (2013) "Mining student repositories to gain learning analytics. An experience report," In IEEE Global Engineering Education Conference EDUCON, 13-15 March, Berlin, Germany, p. 1249-1254.

Rodríguez-Triana, M. J. et al. (2018) “The teacher in the loop: customizing multimodal learning analytics for blended learning". In 8th Int. Conference on Learning Analytics and Knowledge LAK, 07-09 March, Sydney, Australia, p. 417-426.

Santos, J. L., Govaerts, S., Verbert, K. and Duval, E. (2012) "Goal-oriented visualizations of activity tracking: a case study with engineering students". In 2nd Int. Conference on Learning Analytics and Knowledge LAK, April 29-May 02, Vancouver, Canada, p. 143-152. S. B. Shum, D. Gasevic, and R. Ferguson (Eds.)

Shimada, A., Konomi, S. and Ogata, H. (2018) "Real-time learning analytics system for improvement of on-site lectures". In Interactive Tech. and Smart Ed, v.15 n.4, p. 314-331.

Siemens, G. and Baker, R. S. J.d. (2012) "Learning analytics and educational data mining: towards communication and collaboration". In 2nd Int. Conf. on Learning Analytics and Knowledge LAK, April 29-May 02, Vancouver, Canada, p. 252-254. S. B. Shum, D. Gasevic and R. Ferguson (Eds.).

Taibi, D., Bianchi, F., Kemkes, P. and Marenzi, I. (2018) "Learning Analytics for Interpreting". In 10th International Conference on Computer Supported Education CSEDU, 15-17 March, Madeira, Portugal, v. 1, p. 145-154.

Trausan-Matu, S., Dascalu, M. and Rebedea, T. (2014) "PolyCAFe-automatic support for the polyphonic analysis of CSCL chats". In International Journal of ComputerSupported Collaborative Learning, v.9 n. 2, p. 127-156.

Yassine, S., Kadry, S. and Sicilia, M. A. (2016) "A framework for learning analytics in moodle for assessing course outcomes". IEEE Global Engineering Education Conference EDUCON, 10-13 April, Abu Dhabi, UAE, p. 261-266. 